\title{
Caracterización de defectos de superficie en estructuras coladas para prótesis dentales en aleación de cobalto cromo
}

\author{
Josefina Mosquera-Palomino*, MSc. (c), Sonia Elena Pineda-Higuita, MSc. ${ }_{1}$, \\ Carlos Ignacio Vélez- Gómez, Tec $_{1}$, Sebastián Restrepo-Holguín, Est lab 1
}

${ }_{1}$ Grupo de Investigación en Salud y Comunidad, Facultad de Odontología, Programa de Laboratorio de Prótesis Dental, Fundación Universitaria Autónoma de las Américas, Medellín, Colombia

Recibido: 11 de agosto del 2016 Aprobado: 26 de octubre del 2016

*Autor de correspondencia: Josefina Mosquera-Palomino. Fundación Universitaria Autónoma de las Américas, Medellín, Colombia. Calle 34 A N. 76-35. Teléfono (54) 4114848 ext. 2411. Correo electrónico: josefina.mosquera@uam.edu.co

Cómo citar este artículo: Mosquera-Palomino J, Pineda-Higuita SE, Vélez- Gómez Cl, Restrepo-Holguín S. Caracterización de defectos de superficie en estructuras coladas para prótesis dentales en aleación de cobalto cromo. Rev Nac Odontol. 2017;13(24):55-63. doi: http://dx.doi.org/10.16925/od.v12i24.1663

Resumen. Introducción: las aleaciones dentales de cobalto cromo son empleadas en la elaboración de las estructuras coladas que soportan las prótesis dentales. Las fallas que se presentan durante el proceso de colado y acabado de la estructura dan origen a defectos de superficie que afectan las propiedades mecánicas de estas prótesis. Objetivo: caracterizar los defectos de superficie en las estructuras coladas para prótesis dentales en aleación de cobalto cromo, por microscopía electrónica de barrido. Métodos: se realizó un estudio experimental, en el cual se seleccionaron, a conveniencia, 19 estructuras coladas elaboradas en aleación cobalto cromo. En el proceso de colado se tuvieron en cuenta las siguientes variables: presentación de la aleación, tipo de centrífuga, tipo de pulido, brillado y arenado de la estructura. Resultados: las principales características observadas mediante el análisis por microscopio electrónico de barrido (SEM), en las estructuras coladas para prótesis dentales en aleación de cobalto cromo fueron: irregularidades, heterogeneidad superficial, grietas y presencia de carbono. Se encontró una asociación estadísticamente significativa entre la forma de presentación de la aleación y el pulido con brillo y sin brillo, con valor de $p(0,04)$. Conclusiones: la caracterización por microscopía electrónica de barrido permite detectar defectos de superficie que dan origen a fallas mecánicas y deterioros con el uso a mediano y largo plazo, lo cual afecta la calidad de las prótesis dentales.

Palabras claves: aleación, aleación de cobalto cromo, prótesis dental, prótesis parcial removible, técnica de colado dental. 


\title{
Characterization of surface defects in cast structures for dental prostheses in cobalt chromium alloy
}

\begin{abstract}
Introduction: Chromium cobalt dental alloys are used in the preparation of cast structures that support dental prostheses. Failures occurring during the process of casting and finishing of the structure give rise to surface defects that affect the mechanical properties of these prostheses. Objective: To characterize the surface defects in cast structures for dental prostheses in cobalt chromium alloy through scanning electron microscopy. Methods: An experimental study was carried out, in which 19 cast structures prepared in cobalt chromium alloy were conveniently selected. In the casting process, the following variables were taken into account: aspect of the alloy, type of centrifuge, type of polishing, polishing and sandblasting of the structure. Results: The main characteristics observed through the scanning electron microscope (SEM) analysis in cast structures for dental prostheses in cobalt chromium alloy were irregularities, surface heterogeneity, cracks and presence of carbon. A statistically significant association was found between aspect of the alloy and polishing with shine and no shine, with a value of $p(0.04)$. Conclusions: Characterization by scanning electron microscopy allows to detect surface defects that give rise to mechanical failures and deterioration with medium and long term use, which affects the quality of dental prostheses.
\end{abstract}

Keywords: alloy, chromium cobalt alloy, dental prosthesis, removable partial denture, dental casting technique.

\section{Caracterização de defeitos de superfície em estruturas coladas para próteses dentárias em liga de cobalto-cromo}

Resumo. Introdução: as ligas dentárias de cobalto-cromo são empregadas na elaboração das estruturas modeladas que dão suporte às próteses dentárias. As falhas que se apresentam durante o processo de modelagem e acabamento da estrutura dão origem a defeitos de superfície que afetam as propriedades mecânicas dessas próteses. Objetivo: caracterizar os defeitos de superfície nas estruturas modeladas para próteses dentárias em liga de cobalto-cromo por microscopia eletrônica de varredura. Métodos: foi realizado um estudo experimental, no qual foram selecionadas, por conveniência, 19 estruturas modeladas elaboradas em liga de cobalto-cromo. No processo de modelagem, levou-se em consideração as seguintes variáveis: apresentação da liga, tipo de centrífuga, tipo de polimento, brilho e jateamento de areia da estrutura. Resultados: as principais características observadas mediante a análise por microscópio eletrônico de varredura (SEM) nas estruturas modeladas para próteses dentárias em liga de cobalto-cromo foram: irregularidades, heterogeneidade superficial, fendas e presença de carbono. Encontrou-se uma associação estatisticamente significativa entre a forma de apresentação da liga e o polimento com brilho e sem brilho, com valor de $p(0,04)$. Conclusões: a caracterização por microscopia eletrônica de varredura permite detectar defeitos de superfície que dão origem a falhas mecânicas e deteriorações com o uso a médio e longo prazo, o qual afeta a qualidade das próteses dentárias.

Palavras-chaves: liga, liga de cobalto-cromo, prótese dentária, prótese parcial removível, técnica de modelagem dentária. 


\section{Introdución}

Las estructuras coladas en aleaciones de cobalto cromo hacen parte de los diferentes tipos de prótesis dentales, tales como lo son prótesis fijas, removibles y fijas-removibles [1]. Este tipo de aleaciones son de las más utilizadas por sus óptimas propiedades mecánicas, su biocompatibidad oral y costos. Al realizar el colado de una aleación dental, se pueden presentar diversas fallas en la superficie externa e interna de la aleación, debido a múltiples causas, entre ellas no cumplir con los protocolos establecidos para su fabricación, la mala calidad de los materiales utilizados y la falta de destreza del operador. Los efectos principales son: defectos macroscópicos tales como poros, grietas, desniveles, colados incompletos y fragilidad [2]. Defectos microscópicos tales como daños en la microestructura del colado, solubilidad de los metales, tamaño de grano no uniforme, huecos en la estructura atómica, o bien átomos no alineados en un mismo plano. Los defectos mencionados ocasionan diversas fallas estructurales.

La aleación de cobalto cromo utilizada para el presente estudio tiene la siguiente composición: $63 \%$ de cobalto; $27 \%$ de cromo; $6 \%$ de molibdeno; y en proporción menor del $1 \%$ tiene $\mathrm{Fe}, \mathrm{Ni}, \mathrm{Si}$ y Mn. Cada uno de estos metales cumple una función dentro de la aleación: el cobalto incrementa el modulo elástico, aporta resistencia y dureza; el cromo protege contra la pigmentación, la oxidación y la corrosión bucal; el molibdeno confiere dureza y fineza de grano; el níquel aporta ductilidad; y el silicio y el manganeso aumentan la fluidez de la aleación, facilitando el proceso de colado [2].

Las aleaciones de cobalto cromo tienen excelentes propiedades mecánicas. El módulo elástico, el límite proporcional y el límite elástico son los más altos de todas las aleaciones utilizadas en odontología, lo cual significa que son aleaciones rígidas, no se deforman permanentemente por acción de tensiones mecánicas, la alta elongación y la resistencia a la tracción, y garantizan que los ganchos de una prótesis parcial removible no se fracturen con el uso. La baja densidad hace posible conseguir estructuras livianas con espesores mucho más finos, así como un menor volumen de las prótesis, brindando mayor comodidad a los pacientes [3].

El proceso de colado de una aleación dental consiste en fundir y centrifugar a un molde, el cual se construyó previamente en cera; al evaporar la cera se deja el espacio libre para que al entrar el metal y solidificar, copie la estructura que hay dentro del molde. La realización de la estructura colada requiere seguir un protocolo establecido para ello, es decir, seguir un paso a paso de cómo se debe hacer el procedimiento y los aspectos más relevantes a tener en cuenta, como son: composición y calidad de la aleación, tipo de revestimiento, ubicación de bebederos en la estructura metálica, ubicación de anillos en el horno, temperatura de fusión, presión del gas y oxígeno al fundir, clase de máquina para centrifugar el metal fundido, y acabado y pulido de la estructura colada. Si no se controlan estos aspectos se pueden presentar diversas fallas en la superficie interna y externa, así como en la estructura cristalina de la aleación metálica que se forma cuando se deja enfriar esta desde su estado fundido, proceso denominado "solidificación", el cual da lugar a la formación de la red cristalina de la estructura colada, o sea, a la disposición que tienen los átomos en el interior de la estructura $[1,2,4]$.

Las propiedades mecánicas de las aleaciones dentales son el resultado del proceso de elaboración de la estructura protésica colada y conducen a distintas microestructuras en la red cristalina. Los parámetros de colado tienen un efecto significativo sobre las propiedades mecánicas de las aleaciones. La estructura interna y externa de las estructuras coladas puede ser evaluada por diferentes técnicas metalográficas y ensayos mecánicos, entre ellas la microscopía óptica (MO), la microscopía electrónica de barrido (SEM), complementada con prueba de microanálisis (EDS WDS) [5-7].

El objetivo de esta investigación fue caracterizar los defectos de superficie en las estructuras coladas para prótesis dentales, en aleación de cobalto cromo por microscopía electrónica de barrido.

\section{Materiales y métodos}

Se realizó un estudio experimental, en el cual se seleccionaron a conveniencia 19 estructuras metálicas coladas en aleación de cobalto cromo de 3 mm de largo x $1 \mathrm{~mm}$ de ancho, tipo acker, la cual se puede describir como una estructura libre de conector mayor, y con presencia de dos retenedores para el remplazo de una o dos piezas a rehabilitar 
únicamente. La elaboración de estas estructuras se realizó siguiendo el protocolo de la técnica de cera perdida. De forma muy general, esta técnica consiste en un paso a paso en el que se ejecutaron los siguientes procedimientos: diseño de la estructura, duplicado en agar, revestido con revestimiento de fosfato y eliminación de la cera siguiendo una curva de temperatura hasta alcanzar los $950{ }^{\circ} \mathrm{C}$. Luego, se procedió con el colado, el cual se realizó a $950{ }^{\circ} \mathrm{C}$, en un tiempo estimado de fundición de dos minutos, con presiones de 40 psi oxígeno y 15 psi de gas. El paso siguiente fue decapar el colado con una pinza, se arenó con oxido de aluminio de tamaño de partícula de 10um a una presión de seis bares, y se pulió luego la estructura con piedras (rosadas, azules y blancas); esto permite dejar las superficies lisas, ganchos estéticos y el asentamiento de la misma. Acto seguido, se procedió a brillar usando fieltros y tiza chrom, y finalmente se lavaron las estructuras obtenidas.

En este estudio, las variables del proceso que se tuvieron en cuenta fueron las siguientes: presentación de la aleación (lingotes y lágrimas), tipo de centrífuga (manual y eléctrica), pulido (con choque térmico y sin choque térmico), arenado y brillado de la estructura. Una vez obtenida la estructura, se desinfectó con alcohol al 70\% para eliminar residuos de grasa, luego se procedió a analizarlas por microscopía electrónica de barrido SEMEDS, método ASTM E1508-12 ASTM E766-14. Con el equipo microscopio electrónico de barrido (termoiónico), se obtuvieron imágenes con aumentos de varios tamaños, como, por ejemplo, $\mathrm{x} 45 \mathrm{y}$ $500 u m, x 500$ y $50 u m, x 200$ y $100 u m$, entre otras (véanse la figura 1), las cuales permitieron observar diferentes defectos de superficie.

El proyecto fue aprobado por el comité central de investigación de la Fundación Universitaria Autónoma de las Américas y fue considerado como investigación de riesgo mínimo (Código de aceptación P58-2015).

Se realizó un análisis descriptivo de las variables del proceso de colado y de las características observadas en el microscopio electrónico de barrido, así como un análisis bivariado entre las variables de interés. Para dichos análisis se utilizó el programa estadístico PRISMA.

\section{Resultados}

La tabla 1 resume los resultados de las variables tenidas en cuenta en el proceso de elaboración de 19 estructuras coladas tipo acker, en aleación de cobalto cromo para prótesis dentales.

Tabla 1. Características de las variables del proceso de colado

\begin{tabular}{|l|l|c|c|}
\hline \multicolumn{1}{|c|}{$\begin{array}{c}\text { Nombre de } \\
\text { la variable }\end{array}$} & \multicolumn{1}{|c|}{ Categorías } & $\begin{array}{c}\text { Frecuencia } \\
(\mathbf{n})\end{array}$ & $\%$ \\
\hline $\begin{array}{l}\text { Presentación } \\
\text { de la aleación }\end{array}$ & Lingotes & 6 & 31,6 \\
\cline { 2 - 4 } & Lágrimas & 13 & 68,4 \\
\hline $\begin{array}{l}\text { Tipo de } \\
\text { centrífuga }\end{array}$ & Manual & 10 & 53 \\
\cline { 2 - 4 } & Eléctrica & 9 & 47 \\
\hline \multirow{2}{*}{ Tipo de pulido } & Con choque térmico & 10 & 52,6 \\
\cline { 2 - 4 } & Sin choque térmico & 8 & 42,1 \\
\hline \multirow{2}{*}{ Brillo } & Sí & 11 & 57,9 \\
\cline { 2 - 4 } & No & 7 & 36,8 \\
\hline \multirow{2}{*}{ Arenado } & Sí & 7 & 37 \\
\cline { 2 - 4 } & No & 12 & 63 \\
\hline
\end{tabular}

Fuente: grupo investigador

El análisis de las estructuras coladas mediante microscopía electrónica de barrido permitió detectar imperfecciones que no tienen un alcance visual, ya que en todas ellas se observó un acabado óptimo, pero al ser analizadas se encontraron irregularidades y heterogeneidad superficial, grietas, presencia de carbono y de cuerpos extraños (tabla 2).

El 100\% de las estructuras coladas en aleación cobalto cromo para prótesis dentales analizadas en el presente estudio presentan irregularidades y heterogeneidad superficiales; el 73,7\% presentan grietas; el 68,4\% presentan carbono en los ganchos, y un $11 \%$ presentan cuerpos extraños, tales como hebras.

La prueba de chi ${ }^{2}$ logró determinar una correlación significativa entre la forma de presentación de la aleación y el pulido con brillo y sin brillo, en la que el valor de $\mathrm{p}$ fue de 0,04 . No se encontró correlación significativa entre otras variables del proceso tales como la forma de presentación de la aleación y presencia de grietas, así como en pulido (con choque térmico y sin choque térmico) y presencia de grietas, en las que los valores respectivos de $\mathrm{p}$ fueron 0,08 y 0,8 . 


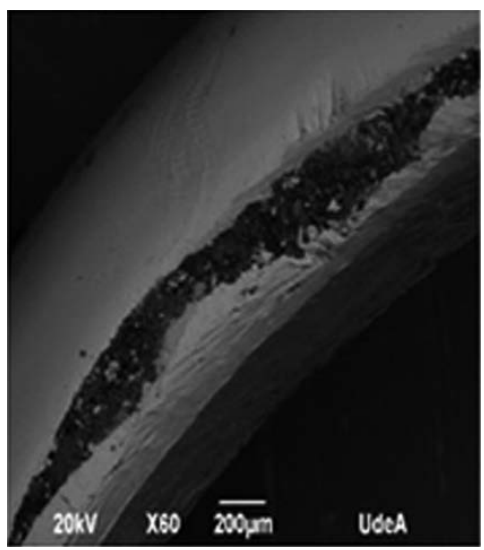

Grietas con incrustación de carbono

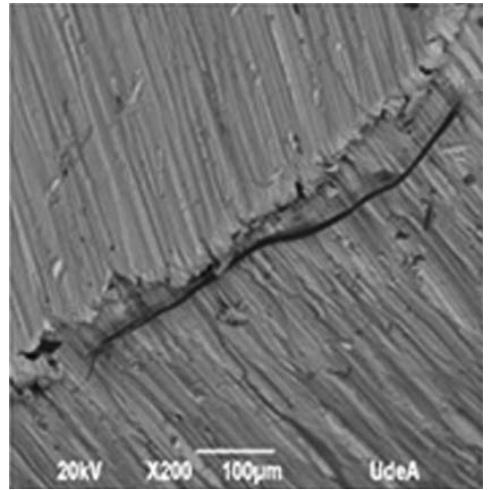

Cuerpo extraño: hebra

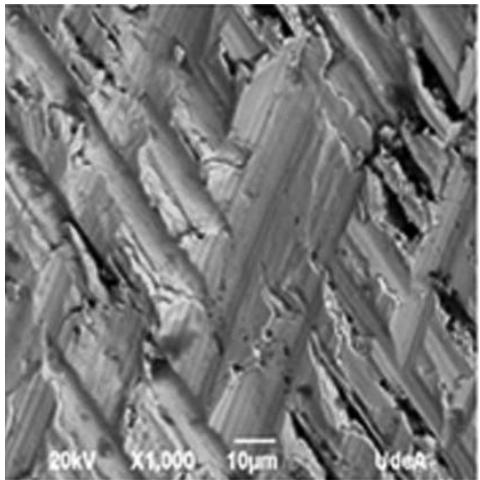

Superficies con múltiples irregulares

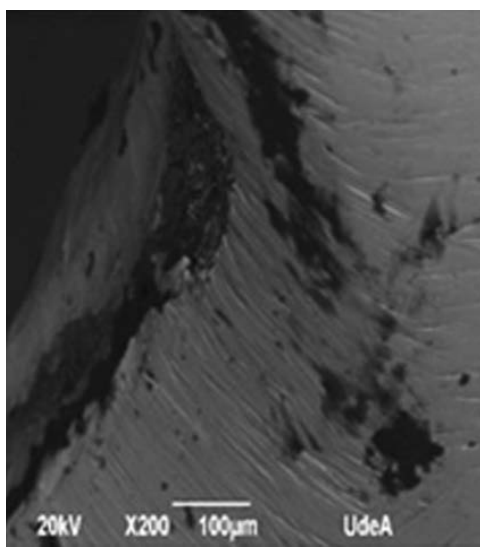

Grietas con incrustación de carbono

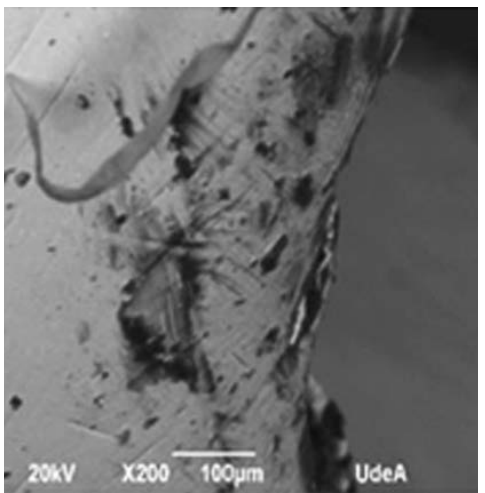

Cuerpo extraño: hebra

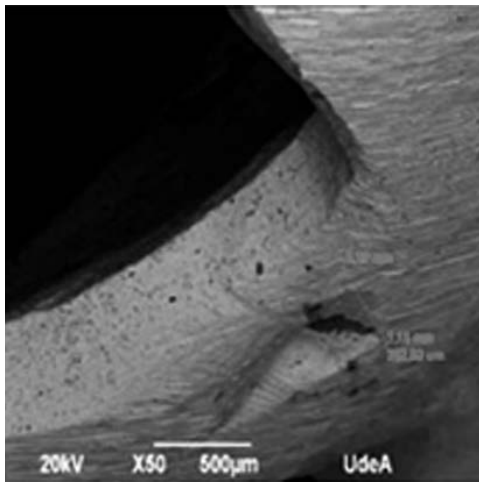

Grietas profundas

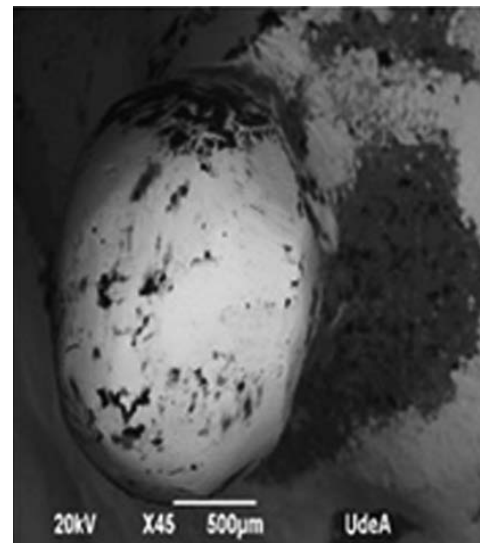

Pin con incrustación de carbono

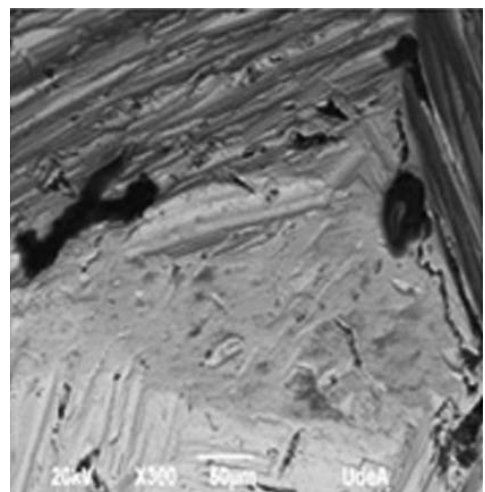

Cuerpos extraños e irregularidades

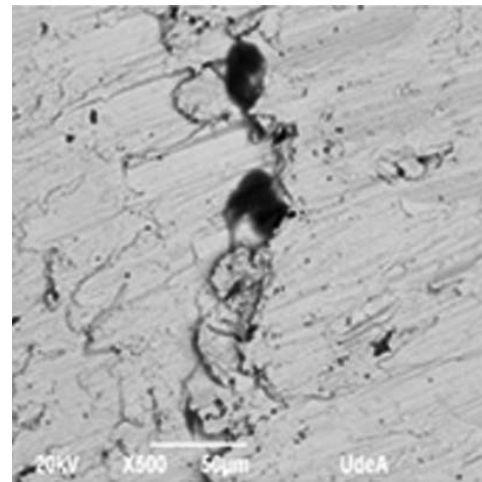

Grietas e irregularidades

Figura 1. Fotografías observadas en el microscopio electrónico de barrido con diferentes aumentos Fuente: grupo investigador 
Tabla 2. Características observadas en el microscopio electrónico de barrido

\begin{tabular}{|c|c|c|c|}
\hline Nombre de la variable & Categorías & $\begin{array}{c}\text { Frecuencia } \\
\%\end{array}$ & $\%$ \\
\hline \multirow{2}{*}{ Presencia de grietas } & Sí & 14 & 73,7 \\
\hline & No & 5 & 26,3 \\
\hline \multirow{2}{*}{$\begin{array}{l}\text { Grietas en brazos } \\
\text { retentivos }\end{array}$} & Sí & 9 & 47,4 \\
\hline & No & 6 & 31,6 \\
\hline \multirow{2}{*}{$\begin{array}{l}\text { Grietas en brazos } \\
\text { recíprocos }\end{array}$} & Sí & 10 & 52,6 \\
\hline & No & 5 & 26,3 \\
\hline \multirow{2}{*}{$\begin{array}{l}\text { Grietas en el Pin de } \\
\text { retención }\end{array}$} & Sí & 1 & 5,3 \\
\hline & No & 14 & 73,7 \\
\hline \multirow{2}{*}{$\begin{array}{l}\text { Irregularidades y hete- } \\
\text { rogeneidad superficiales }\end{array}$} & Sí & 19 & 100 \\
\hline & No & 0 & 0 \\
\hline \multirow{2}{*}{$\begin{array}{l}\text { Irregularidades y hete- } \\
\text { rogeneidad superficiales } \\
\text { en brazos retenedores }\end{array}$} & Sí & 15 & 78,9 \\
\hline & No & 0 & 0 \\
\hline \multirow{2}{*}{$\begin{array}{l}\text { Irregularidades y hete- } \\
\text { rogeneidad superficiales } \\
\text { en el pin de retención y } \\
\text { cajuela }\end{array}$} & Sí & 13 & 68,4 \\
\hline & No & 2 & 10,5 \\
\hline \multirow{2}{*}{$\begin{array}{l}\text { Presencia de carbono } \\
\text { en los brazos de rete- } \\
\text { nedores }\end{array}$} & Sí & 13 & 68,4 \\
\hline & No & 2 & 10,5 \\
\hline \multirow{2}{*}{$\begin{array}{l}\text { Presencia de carbono } \\
\text { en el pin de retención y } \\
\text { cajuela }\end{array}$} & Sí & 8 & 42,1 \\
\hline & No & 7 & 36,8 \\
\hline \multirow{2}{*}{$\begin{array}{l}\text { Presencia de cuerpos } \\
\text { extraños }\end{array}$} & Sí & 2 & 11 \\
\hline & No & 17 & 89 \\
\hline
\end{tabular}

Fuente: grupo investigador

\section{Discusión}

Las propiedades mecánicas de las aleaciones son el resultado del proceso de elaboración que conduce a distintas microestructuras. Los parámetros de colada tienen un efecto significativo sobre las propiedades mecánicas de las aleaciones coladas. Estudios similares a este se han realizado en diferentes partes del mundo y en estos la estructura interna de las aleaciones coladas puede ser evaluada por diferentes técnicas metalográficas y ensayos mecánicos; estos últimos, como lo son, por ejemplo, las inspecciones radiográficas, la microscopía óptica (MO), la microscopía electrónica de barrido (SEM), complementadas con prueba de microanálisis (EDS WDS) [5-7].

La inspección radiográfica se ha utilizado para identificar la presencia de discontinuidades en las estructuras de prótesis parciales removibles $[5,8]$. La uniformidad en los elementos que constituyen la aleación y las propiedades mecánicas, constituye un índice de calidad y es una de las características más importantes a exigir de una aleación a fin de ser utilizada como estructura metálica, junto con la ausencia de heterogeneidades perjudiciales para una adecuada manipulación del mismo y un comportamiento apropiado en la función que deba desempeñar, de manera que la estructura metálica de cromo-cobalto responda por igual a los procesos a que será sometida cuando esté en uso, es decir, igual grado de confiabilidad [5].

Las aleaciones de cobalto basadas en el sistema Co-Cr-Mo se utilizan para componentes de reemplazos articulares desde hace tiempo, debido a su excelente resistencia a la abrasión y a la corrosión, así como a sus buenas propiedades mecánicas. El principal problema que presenta esta aleación es su baja ductilidad y la baja resistencia a la fatiga del metal colado.

Las propiedades mecánicas de esta aleación dependen, principalmente, de características microestructurales tales como la cantidad, la distribución y la morfología de fases duras que dependen de las condiciones de procesado utilizadas. Los implantes producidos por colado se obtienen por el método de la cera perdida. La desventaja del metal colado es que la pieza queda con porosidad, no presenta homogeneidad química y tenga una estructura de solidificación dendrítica con precipitaciones de carburos que la hace frágil, poco dúctil y con baja resistencia a la fatiga [6].

En otro estudio se analizó la microestructura de las aleaciones dentales comerciales TI-6AL-4V y Ni 65\%-CR 22,5\%-MO 9,5\%, mediante microscopía electrónica de barrido (SEM), y microanálisis por técnica de espectrometría de energías dispersivas de rayos $\mathrm{x}$ (EDS). Se encontró que durante el proceso de colado, se produce una unión química con presencia de oxígenos entre estas dos aleaciones, evidenciando estructuras dendríticas heterogéneas a lo largo de las muestras debido a la irregularidad en el espesor de las barras utilizadas y los precipitados en sus fronteras de Mo. El estudio concluye que las barras muestran irregularidades, rugosidades, porosidad y heterogeneidad en su superficie que evidencian fallas durante el proceso de elaboración de la pieza, las cuales podrían llegar a afectar su comportamiento mecánico y de resistencia a la corrosión $[9,10]$. 
Se presentan cambios en las microestructuras de la aleación de Cr-Co después de sucesivos calentamientos, lo que indica que se presentan cambios en sus propiedades mecánicas [11]. También hay estudios sobre caracterización microestructural de aleaciones coladas de aluminio, mediante análisis metalográfico utilizando microscopía óptica (MO) y microscopía electrónica de barrido (SEM), complementado con técnicas de microanálisis EDS y wDS, las cuales permitieron observar el crecimiento de las dendritas y el porcentaje de porosidades internas [12].

Mediante SEM, se ha demostrado que las exposiciones a altas temperaturas durante la fundición del metal pueden dar lugar a precipitados, los cuales afectan la resistencia de la aleación y causan fragilidad [13].

Las microestructuras de la aleación Cr-Co-Mo, fabricadas utilizando la fusión selectiva por láser (SLM), también han sido objeto de estudio. Se encontró que la formación de defectos microestructurales es difícil de evitarse durante el proceso de SLM. Por su parte, las aleaciones de Co Cr Mo, elaboradas por esta técnica, pueden conseguir propiedades mecánicas mejoradas, lo que implica que son "defecto tolerante" [14].

Aleaciones dentales fabricadas en Ni-Co Cr W, por fusión selectiva con láser (SLM), fueron analizadas con om y SEM. Se observó la microestructura, la dureza, las propiedades mecánicas, el comportamiento electroquímico y la liberación de metal [15].

Se elaboraron probetas de aleación dental de cobalto cromo por tres técnicas diferentes: casting (CST), molienda (MIL) y sinterización selectiva por láser (SLM). La porosidad de los tres grupos se evaluó usando escáneres de rayos $\mathrm{x}$, y las microestructuras se evaluaron mediante examen SEM, EDX. Se encontró que las probetas con mayor porosidad fueron las fabricadas mediante fundición del metal, además, los tres grupos tenían diferentes microestructuras, lo cual significa que la microestructura de las aleaciones dentales en cobalto cromo dependen de la técnica de fabricación empleada [16].

La resistencia a la tracción, el alargamiento, la microdureza, la composición y la microestructura de dos aleaciones a base de níquel cromo fundido bajo diferentes condiciones de colado fue evaluada mediante examen SEM. Se encontró que las propiedades de la aleación tienen relación con la técnica de fundición empleada [17].
El tratamiento térmico de la aleación influye en la microestructura y las propiedades mecánicas de la aleación como lo referencia el estudio de Mengucci, Barucca, Gatto, Bassoli, Denti et al. [18], en el cual se evaluó la respuesta mecánica de la aleación y la microestructura en el estado sinterizado. Luego de tratamientos térmicos posteriores a la fabricación, se realizaron mediciones de rugosidad y dureza, de resistencia a la tracción y ensayos de flexión, a fin de estudiar la respuesta mecánica de la aleación, mientras que la difracción de rayos $\mathrm{x}$ (XRD), microscopía electrónica (SEM, TEM, STEM), técnicas y microanálisis (EDX), se utilizaron para investigar la microestructura en diferentes condiciones.

Muchos laboratoristas dentales utilizan aleación reciclada para elaborar las prótesis dentales. El impacto de la cantidad de material reciclado en las propiedades mecánicas y la estructura de la aleación dental Co Cr Mo ha sido estudiado y se ha observado que la resistencia mecánica de todas las aleaciones recicladas es peor que la resistencia de la aleación no reciclada, debido a que no hay una estructura uniforme. Por otra parte, se observó la aparición de precipitados de varios tipos de carburos y la presencia de fases intermetálicas, las cuales tienen un efecto determinante sobre las propiedades mecánicas de las piezas [19].

Los aditamentos y materiales de pulido también son un factor determinante en la microestructura de la aleación colada, la biocompatibilidad y las implicaciones clínicas, por eso se han evaluado los efectos del acabado y el pulido de las superficies $[20,21]$.

El contenido de carbono en las estructuras coladas influye en la microestructura, ya que se han determinado los efectos de la concentración de carbono en estas y en las propiedades de tracción de las aleaciones dentales. La microscopía electrónica de barrido (SEM) y la sonda de electrones para microanálisis (EPMA) revelaron los precipitados que se formaron en todas las muestras de aleación y los cambios de fase que se dieron en la microestructura [22].

El presente estudio permitió observar, a través del análisis de microscopía electrónica de barrido, cómo las estructuras coladas tipo acker en aleación de cobalto cromo para prótesis dentales no poseen una superficie uniforme, sino mucha heterogeneidad manifiesta en múltiples irregularidades superficiales, grietas, además de alta presencia de 
carbono que se incrusta en estas, y en dos estructuras se hallaron cuerpos extraños como hebras. Resultados muy similares a los estudios citados anteriormente, lo cual tiene un efecto en la microestructura de la aleación colada y la hace susceptible a fallas mecánicas durante el uso.

La revisión de literatura científica especializada citada anteriormente y los resultados del presente estudio permiten afirmar que los fracasos clínicos en las prótesis removibles se asocian, principalmente, con la exactitud y la precisión del diseño, y los procedimientos de laboratorio tales como encerado, revestido, fusión y técnica de fundición, pulido y acabado de la estructura, así como con la calidad de la aleación.

\section{Conclusiones}

Todas las estructuras analizadas por microscopía electrónica de barrido presentan irregularidades y heterogeneidad superficiales, alto porcentaje de grietas y presencia de carbono, de manera que contribuyen a que se presenten fallas mecánicas y de biocompatibilidad que afectan la calidad de las prótesis dentales, así como conllevan a deterioros con el uso a mediano y largo plazo.

La caracterización por microscopía electrónica de barrido permite detectar defectos superficiales en las estructuras coladas que no tienen un alcance visual.

Los resultados obtenidos indican que en una técnica de elaboración tan manual de las estructuras coladas, se requiere revisar continuamente el protocolo de elaboración, la destreza del operador y el mantenimiento de los equipos, a fin de garantizar las propiedades mecánicas de las prótesis dentales y los estándares de calidad en los procesos de rehabilitación dental.

\section{Agradecimientos}

Los investigadores agradecemos a la Fundación Universitaria Autónoma de las Américas por la financiación del proyecto. Al Laboratorio de Materiales de la Universidad de Antioquia, donde se realizó el análisis por microscopio electrónico de barrido. Al estudiante del semillero de investigación del Programa de Laboratorio de Prótesis Dental seILAD de la Fundación Universitaria
Autónoma de las Américas, Juan Sebastián LópezJaramillo, por su apoyo en la elaboración de las estructuras coladas.

\section{Referencias}

[1] Guzmán-Báez, H. Biomateriales odontológicos de

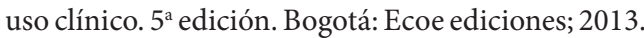

[2] Skinner J. La ciencia de los materiales dentales. $11^{\text {a }}$ edición. España, ELSEVIER; 2004.

[3] Craig, R. Materiales de odontología restauradora. 10a edición, Madrid: Harcourt-Brace; 1998.

[4] Harcourt M. Propiedades de los biomateriales. Materiales de odontología restauradora. $10^{a}$ edición, Madrid: Harcourt-Brace; 1998

[5] Rivas MA, María A. (2001). Evaluación visual y radiográfica de discontinuidades en estructuras metálicas de prótesis parciales removibles. Acta Odontológica Venezolana. 39(2):25-34.

[6] Borrás VA, Paolini A, Ballester JM, Escuder V, Sanchis, FR. Estudio de la influencia de los tratamientos térmicos en la microdureza y microestructura de aleaciones Co-Cr-Mo. Universidad Politécnica de Valencia. Actas vin Congreso Nacional de Propiedades Mecánicas de Sólidos. Gandía; 2002. 487-96.

[7] Fomaro, J. G. Microstructural evolution during solution treatment of Co-Cr-Mo -C biocompatible alloys. 2012;9:47-59. doi: http://dx.doi.org/10.1016/j. matchar.2012.03.006. (http://www.sciencedirect. com/science/article/pii/S1044580312000630

[8] Elamin E, Yahia I, Mohsen A, Tarnjit S. Radiographic detection of porosities in removable partial denture castings. The Journal of Prosthetic Dentistry. 1985;54(5):674-7.

[9] Echeverri, A. Caracterización metalográfica de barras para sobre dentaduras, elaboradas por sobre colado de pilares para implantes dentales. Rev Fac de Od Univ Ant. 2013;25(1):26-43.

[10] Sunder R. Radiographic and metallographic evaluation of porosity defects and grain structure of cast chromium cobal removable partial dentures. T J of Prost Dent. 1993;369-73.

[11] Vaillant AS, Corne P, De March P, Fleutot S, Cleymand F, Jiehl P. Efecct of recasting of cobalt chromium alloy on its mechanical properties, Euro Cell and Mat. 2015;30(5):17.

[12] García J., González C, Houbaert Y, González M. Caracterización microestructural de aleaciones coladas Al-7 Si-Cu Al-7 Si-Ni con adiciones de sr. Rev Lat de Metal y Mat. 2003; 23(2):20-4.

[13] Hae R, Young K, Jun S, Bong K, Kyo-Han K, et al. Comparison of in vitro biocompatibility of a $\mathrm{Co}-\mathrm{Cr}$ 
dental alloy produced by new milling/post-sintering or traditional casting technique. Materials Letters, 2016; 178:300-3. Disponible en: http://www.sciencedirect. com/science/article/pii/S0167577X16307881

[14] Qian B, Saeidi K, Kvetková L, Lofaj F, Xiao C., et al. Aleaciones dentales Co-Cr-Mo. Defectos tolerantes preparados por fusión selectiva por láser. Materiales Dentales. 2015;31(12):1435-44. Disponible en: http://www.sciencedirect.com/science/article/pii/ S0109 564115003991

[15] Anjin L, Songquan W, Yiliang G, Junlei L, Chaoqian $\mathrm{Z}$, et al. Investigación sobre la microestructura, propiedades mecánicas y el comportamiento a la corrosión del selectivo por láser funde la aleación Co Cr w para aplicación dental. Ciencia de los Materiales e Ingeniería. 2015; 49:517-525. Disponible en: http://www.sciencedirect.com/science/article/ pii/ S0928493115000338

[16] Al Jabbari Y, Koutsoukis T, Barmpagadaki X, Zinelis S. Metalúrgica y caracterización de aleaciones dentales interfacial PFM Co-Cr fabricado mediante fundición, de la molienda o de fusión selectiva por láser. Materiales Dentales. 2014;30(4):E79-E88. Disponible en: http://www.sciencedirect.com/science/ article/pii/S0109564114000207

[17] Bauer J, Costa JF, Carvalho CN, Miranda RE, Dourado $\mathrm{A}$, Reis $\mathrm{A}$. Caracterización de dos aleaciones dentales de Ni-Cr y la influencia del modo de colada en las propiedades mecánicas. Diario de Prosthodontic Research. 2012;56(4):264-271. Disponible en: http://www.sciencedirect.com/science/article/ pii/S1883195812000333.
[18] Mengucci P, Barucca G, Gatto A, Bassoli E, Denti $\mathrm{L}$, et al. Efectos de los tratamientos térmicos en la microestructura y mecánico propiedades de una aleación biomédica $\mathrm{Co}-\mathrm{Cr}-\mathrm{Mo}-\mathrm{W}$ producido por sinterización láser. Diario del comportamiento mecánico de materiales biomédicos. 2016;60:10617. Disponible en: http://www.sciencedirect.com/ science/ article/pii/S1751616116000023

[19] Walczak M, Cerveza K, Surowska B, Borowicz J. El tema del uso de aleaciones de Co Cr Mo refundidos en prótesis dentales. Archivos de ingeniería civil y mecánica. 2012;12(2):171-7. Disponible en: http://www.sciencedirect.com/science/article/pii/ S1644966512000301

[20] McGinley EL, Coleman DC, Moran P, Garry JP. Efectos de las condiciones de acabado de superficie sobre la biocompatibilidad de una aleación de fundición de níquel-cromo dental. Dental Materials. 2011;27(7):637-50. Disponible en: http:// www.sciencedirect.com/science/article/pii/S010 9564111000741

[21] Henriques B, Faria S, Soares D, Silva FS. Efecto del calor de presión sobre la resistencia al cizallamiento de la porcelana dental para sustratos de aleación co $\mathrm{Cr}$ Mo si con diferentes tratamientos superficiales. Ciencia de los Materiales e Ingeniería. 2013;33(1):55763. Disponible en: http://www.sciencedirect.com/ science/article/pii/S092849311200464X

[22] Kenta Y, Manami M, Akihiko. Efectos de la concentración de carbono en la microestructura y las propiedades mecánicas de las recién coladas aleaciones libres de níquel-base-28Cr-9W Co dentales. Ciencia de los Materiales e Ingeniería. 2014;40(1):12734. Disponible en: http://www.sciencedirect.com/ science/article/pii/S0928493114001799 